\title{
Complementary Modes of Action of Tissue-type Plasminogen Activator and Pro-urokinase by which Their Synergistic Effect on Clot Lysis May Be Explained
}

\author{
Ralph Pannell, James Black, and Victor Gurewich \\ Vascular Laboratory, Department of Biomedical Research, St. Elizabeth's Hospital and Tufts University School \\ of Medicine, Boston, Massachusetts 02135
}

\begin{abstract}
Tissue plasminogen activator (t-PA) and/or pro-urokinase (pro-UK) induced lysis of standard ${ }^{125} \mathrm{I}$-fibrin clots suspended in plasma was studied. Doses were kept below the concentration at which a nonspecific effect was seen, i.e., where fibrinogenolysis and major plasminogen consumption were observed. Small amounts of t-PA potentiated clot lysis by pro-UK by attenuating the lag phase characteristic of pro-UK, and causing a much earlier transition to the rapid phase of lysis. Similar promotion of the fibrinolytic effect of pro-UK was obtained when clots were pretreated with UK or with a little plasmin ( $<1 \%$ clot lysis). Promotion by plasmin was nullified by a subsequent treatment of the clot with carboxypeptidase $B$, indicating that the plasmin effect was related to the exposure of carboxy terminal lysine residues on fibrin. These lysine termini, absent in undegraded fibrin, are known to be essential for the high affinity binding of plasminogen to fibrin. In contrast, clot lysis by t-PA was unaffected by plasmin pretreatment and little affected by carboxypeptidase $B$ treatment of the fibrin substrate. Therefore, plasminogen bound to lysine termini on fibrin, although found to be essential for pro-UK, did not appear to serve as a substrate for $t-P A$.

Selective activation of fibrin bound plasminogen has been attributed to the conformational change in Glu-plasminogen that occurs as a result of binding. The present findings suggest that this conformational change occurs when plasminogen is bound to a terminal lysine but not to an internal lysine. Plasminogen bound to the latter site on fibrin was activated by t-PA and therefore is involved in the ternary complex. This initiates lysis of the undegraded clot and exposes the plasminogen binding sites required by pro-UK. By their complementary activation of fibrin bound plasminogen, t-PA followed by pro-UK induces efficient and synergistic fibrinolysis, whereas each is relatively inefficient when used alone.
\end{abstract}

\section{Introduction}

Both tissue-type plasminogen activator (t-PA) ${ }^{1}$ and urinarytype single chain plasminogen activator, more commonly

Address reprint requests to Dr. Gurewich, Vascular Laboratory, St. Elizabeth's Hospital, 736 Cambridge Street, Boston, MA 02135.

Received for publication 17 April 1987 and in revised form 20 August 1987.

1. Abbreviations used in this paper: pro-UK, pro-urokinase; t-PA, tissue-type plasminogen activator.

J. Clin. Invest.

(c) The American Society for Clinical Investigation, Inc.

0021-9738/88/03/0853/07 \$2.00

Volume 81, March 1988, 853-859 called pro-urokinase (pro-UK), induce a selective activation of fibrin-bound plasminogen, while sparing free or systemic plasminogen and thus fibrinogen. They differ significantly, however, in the mechanisms by which this fibrin specificity is achieved.

Tissue plasminogen activator binds strongly to fibrin. As a result, a ternary complex with plasminogen bound to a neighboring region on fibrin is formed. This complex enables plasmin generation and fibrinolysis to take place (1). Although this mechanism is well established, the t-PA binding site on fibrin has not been identified.

Although pro-UK was discovered in urine as a result of its binding to fibrin/Celite $(2,3)$, it has been found not to have a significant fibrin clot affinity in a plasma milieu (4). Instead, the fibrin selectivity of pro-UK in plasma has been attributed to its selective activation of fibrin-bound plasminogen (5) due to the conformational change that occurs in Glu-plasminogen when its high affinity lysine binding site is occupied by a lysine residue (6). This conformational change makes it functionally equivalent to Lys-plasminogen, which is far more sensitive to activation by pro-UK than is Glu-plasminogen (7). Alternatively, it has been suggested that fibrin dissociates a complex, made up of pro-UK with a competitive inhibitor, thereby triggering fibrinolysis (8). However, this inhibitor remains to be identified and a pro-UK complex has not been demonstrated.

Some insight into the mechanisms by which pro-UK or t-PA selectively activate fibrin bound plasminogen may be obtained by studying their effects in a model clot lysis system. This has shown that the two activators are synergistic in certain combinations $(9,10)$ suggesting complementary mechanisms of action. However, synergism has been contested by other investigators who failed to find synergism in vitro (11) while finding it in vivo $(12,13)$.

In the present study, the presence of synergism in a plasma milieu in vitro is reevaluated, and an explanation for its apparent occurrence is offered based on the finding that $\mathrm{t}-\mathrm{PA}$ and pro-UK appeared to activate different plasminogens on the fibrin clot.

\section{Methods}

\section{Materials}

Pro-UK was provided by Collaborative Research, Inc. (Lexington, MA). The preparation was $>99 \%$ single chain as determined by amidolytic assay (14). Urokinase was a product of Green Cross (Osaka, Japan), a generous gift from Dr. A. B. Chandler and Dr. M. Speidel, Medical College of Georgia (Augusta, GA). Single-chain t-PA was a gift from Integrated Genetics (Framingham, MA) and was judged by reducing SDS-PAGE to be $>98 \%$ single chain. Human plasmin was purchased from Sigma Chemical Co. (St. Louis, MO). Whole human plasma was obtained from St. Elizabeth's Hospital blood bank. $4 \mathrm{U}$ were pooled and stored frozen at $-20^{\circ} \mathrm{C}$. Bovine serum albumin ("pu- 
rified" grade) was purchased from Calbiochem-Behring (Torry Pines, CA).

Human fibrinogen was a product of Kabi Vitrum (Stockholm, Sweden). Plasminogen was removed by passage of a diisopropylflourophosphate (DFP) treated solution over Lysine-Sepharose equilibrated to $5 \mathrm{mM}$ trisodium citrate, $0.15 \mathrm{M} \mathrm{NaCl}$. The product was then dialyzed against $0.05 \mathrm{M}$ hydroxyethylpiperazinesulfonic acid (Hepes) (pH 7.4), $0.15 \mathrm{M} \mathrm{NaCl}$.

Carboxypeptidase B (porcine pancreatic, DFP treated) was obtained from Sigma Chemical Co. The preparation was $>98 \%$ pure by SDS-PAGE and had no effect on the chromogenic activities of UK or plasmin, or on the activation of plasminogen by UK in buffer. These assays were performed as previously described (5). Unitage for the carboxypeptidase B was taken as provided by the supplier.

\section{Procedure}

Standard clot lysis system. ${ }^{125}$ I-labeled fibrin clots were prepared from plasminogen-free fibrinogen (Kabi, Stockholm, Sweden; $5 \mathrm{mg} / \mathrm{ml}$ ) mixed with ${ }^{125}$ I-labeled human fibrinogen (Ibrin; Amersham Corp., Arlington Heights, IL). Fibrinogen $(0.25 \mathrm{ml})$ was clotted by addition of $10 \mathrm{mM} \mathrm{CaCl}_{2}$ and thrombin (Thrombostat; Parke-Davis Co., Detroit, MI; final $1 \mathrm{NIH} \mathrm{U/ml).} \mathrm{Clots} \mathrm{were} \mathrm{formed} \mathrm{in} 5 \mathrm{~mm}$ (i.d.) glass tubing, incubated for $2 \mathrm{~h}$ at room temperature, and stored refrigerated.

For lysis, clots were expressed intact from the forming tube and suspended in $3 \mathrm{ml}$ of bank citrated plasma with added activators and incubated at $37^{\circ} \mathrm{C}$. Lysis was monitored by taking $50-\mu$ laliquots from the supernatant at time points for counting of released radioactivity. Some clots were specially treated before the lysis experiment as described below.

Treatment of clots with plasmin. Clots were pretreated with plasmin by incubation in $1 \mathrm{ml}$ of plasmin solution $(2 \mu \mathrm{g} / \mathrm{ml}$ in $0.05 \mathrm{M}$ Hepes, pH 7.4, $0.15 \mathrm{M} \mathrm{NaCl}, 1 \mathrm{mg} / \mathrm{ml}$ bovine serum albumin) for 0-30 min at $37^{\circ} \mathrm{C}$. Plasmin was inactivated by incubation of the clots in $1 \mathrm{ml}$ of plasma for $30 \mathrm{~min}$ at $25^{\circ} \mathrm{C}$. The degree of digestion was estimated from the amount of radioactivity released.

Treatment of clots with carboxypeptidase B. Some plasmin treated clots were subsequently treated with carboxypeptidase $B(1 \mathrm{U} / \mathrm{ml}$ in $0.05 \mathrm{M}$ Hepes, pH 7.8, $1 \mu \mathrm{M} \mathrm{ZnCl}_{2}, 0.15 \mathrm{M} \mathrm{NaCl}, 1 \mathrm{mg} / \mathrm{ml}$ bovine serum albumin) for $60 \mathrm{~min}$ at $37^{\circ} \mathrm{C}$. All clots were washed extensively in citrate saline.

Determination of lysine released by carboxypeptidase $B$ from plasmin-treated fibrin. Fibrin clots $(1 \mathrm{ml}, 2 \mathrm{mg} / \mathrm{ml})$ were treated first with plasmin as described above (except without albumin) and then washed exhaustively in Hepes-buffered-saline. The clots were then treated with carboxypeptidase B as described but without albumin and for $2 \mathrm{~h}$. This supernatant was then withdrawn for amino acid analysis. Controls were run with (1) clots treated with carboxypeptidase B but not pretreated with plasmin, and (2) clots pretreated with plasmin and incu- bated in parallel but without the addition of carboxypeptidase B. Amino acid analysis was performed by the Microchemistry Facility of the Harvard Biological Laboratories (Cambridge, MA).

Examination of maximal rates of fibrin specific lysis. Fibrin specificity was defined as complete clot lysis without degradation of fibrinogen or loss of plasminogen. In the standard system lysis was induced by a dose-response series of each activator: pro-UK, $200-600 \mathrm{ng} / \mathrm{ml}$; and t-PA, $30-250 \mathrm{ng} / \mathrm{ml}$. The threshold of specificity was judged as the concentration just below that which showed a significant loss in plasminogen and fibrinogen in the plasma at the end of lysis. For this series, $5 \mathrm{ml}$ of plasma was used in order to dilute the potentiating effect of fibrin degradation products on nonspecificity. Plasminogen was assayed as described previously (5). Fibrinogen was assayed as thrombin clottable protein. The rate of lysis (in percent lysis/hour) was calculated for the linear portions of the lysis curves in each case. Lysis rates were also determined for a range of combinations of the two activators that preserved specificity.

\section{Results}

At a concentration of $300 \mathrm{ng} / \mathrm{ml}$, pro- $\mathrm{UK}$ induced clot lysis, which was characterized by a lag phase in which $<10 \%$ lysis occurred in the first $90 \mathrm{~min}$, at which time there was an abrupt acceleration to a linear rate of $\sim 20 \%$ /hour until the fibrin substrate remaining became rate limiting. The addition of 100 $\mathrm{ng} / \mathrm{ml}$ of UK (which by itself induced no appreciable lysis) promoted pro-UK induced lysis dramatically. Most notable was the shortening of the lag phase and the earlier establishment of the rapid linear rate of lysis. Consequently, lysis at 90 min was nearly $50 \%$ complete. Similar results were obtained when the activators were used sequentially rather than in simultaneous combination. When clots were treated for one hour with UK in plasma, washed in fresh plasma to allow for inhibition of plasmin, and transferred to fresh plasma containing pro-UK, marked potentiation of pro-UK induced clot lysis was again seen (Fig. $1 \mathrm{~A}$ ).

At a concentration of $50 \mathrm{ng} / \mathrm{ml}$, t-PA induced clot lysis that was comparable overall to that induced by $300 \mathrm{ng} / \mathrm{ml}$ pro-UK, but proceeded at a very different rate. When the activators were combined, clot lysis was promoted to an extent greater than an additive effect. (The combination induced $50 \%$ clot lysis equivalent to $>250 \mathrm{ng} / \mathrm{ml}$ t-PA or $>600 \mathrm{ng} / \mathrm{ml} \mathrm{pro-UK}$ [Figs. $1 B$ and 5.])

When $50 \mathrm{ng} / \mathrm{ml} \mathrm{t-PA}$ in plasma was used as a pretreatment for clots, followed by extensive washing and transfer to fresh

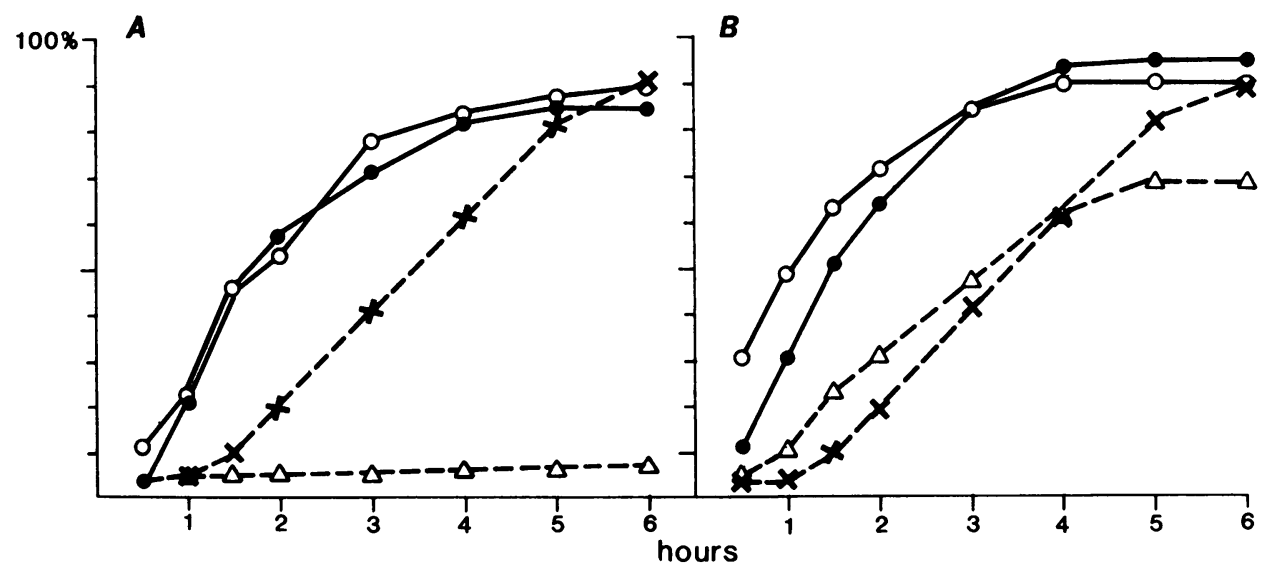

Figure 1. ${ }^{125}$ I-fibrin clot lysis in plasma induced by pro-UK alone and in simultaneous and sequential combinations with UK and t-PA. (A) pro-UK, $300 \mathrm{ng} / \mathrm{ml}(\times)$; UK, $100 \mathrm{ng} / \mathrm{ml}(\triangle)$; simultaneous proUK plus UK (๑); clots pretreated for $1 \mathrm{~h}$ with $\mathrm{UK}$ in plasma, followed by incubation with pro-UK (O); $(B)$ pro-UK, $300 \mathrm{ng} / \mathrm{ml}(\times)$; t-PA, 50 $\mathrm{ng} / \mathrm{ml}(\Delta) ;$ simultaneous pro-UK plus t-PA (๑); clots pretreated for 1 $h$ with t-PA in plasma, followed by incubation with pro-UK (O). 
plasma containing $300 \mathrm{ng} / \mathrm{ml}$ pro-UK, a similar promotive effect on subsequent lysis by pro-UK was seen (Fig. $1 B$ ). By contrast, when similar pretreatment with t-PA was followed by transfer to fresh plasma containing more t-PA at the same concentration, there was no promotive effect (not shown).

The feature common to the action of UK and t-PA was the nearly immediate onset of lysis and thus an early degree of plasmic modification of the fibrin surface. To examine the contribution of this factor, clots were pretreated with plasmin itself in buffer for various times up to $30 \mathrm{~min}$ and then incubated in plasma to inhibit the plasmin. Less than $1 \%$ clot lysis was induced by this pretreatment, based on the amount of radioactivity released into the supernatant. There was again a dramatic increase in susceptibility to pro-UK induced lysis compared with an untreated clot. In contrast, there was no such influence of plasmin pretreatment of clots on the course of t-PA-induced clot lysis (Fig. 2).

The effect of plasmin treatment of the fibrin clots on proUK-induced lysis could be completely abolished by an intervening treatment with carboxypeptidase $B$, which removes the carboxy-terminal basic amino acids from the plasmin cleaved fragments at the fibrin surface. In contrast, t-PA induced clot lysis was insignificantly affected since lysis of untreated, plasmin treated, or carboxypeptidase B-treated clots was comparable (Fig. 3).

The release of lysine by carboxypeptidase B treatment of plasmin treated fibrin (1-2\% digested) was shown by amino acid analysis of a supernatant after incubation. There was a yield of $\sim 0.4$ nmol lysine above the control background. Considering three lysine termini of a half-degraded fibrin monomer, this is equivalent to $45 \mu \mathrm{g}$ of fibrin or about $2 \%$ of the fibrin available.

Carboxypeptidase B had an inhibitory effect when added directly to the plasma clot lysis mixture rather than used as a pretreatment. The induction of lysis by t-PA, pro-UK, and by UK were all sensitive to the presence of carboxypeptidase B (not shown). Thus great care was taken to exhaustively wash fibrin clots in the carboxypeptidase B pretreatment experiments to fully extract the enzyme in order to eliminate any continuing effect on any elements of the fibrinolytic system. That washing was sufficient was evidenced by the absence of inhibition by carboxypeptidase B pretreatment on t-PA induced fibrinolysis, whereas inhibition regularly occurred when carboxypeptidase B was present.

Synergism was most pronounced when carboxypeptidase B treated clots were used. The combination of t-PA and pro-UK overcame the extreme retarding effect of carboxypeptidase B pretreatment of the clots on pro-UK induced lysis. Though lysis by pro-UK was $<10 \%$ in $5 \mathrm{~d}$ and lysis by t-PA at this low dose $(10 \mathrm{ng} / \mathrm{ml})$ was only $50 \%$ in $5 \mathrm{~d}$, lysis induced by the combination was complete within $1 \mathrm{~d}$ (Fig. 4).

The maximal rate of fibrin specific lysis unaccompanied by a significant loss of plasminogen or fibrinogen was determined for pro-UK and for t-PA. The dose-response series showed that the lag phase for pro-UK persisted even at the maximal specific dose. By contrast, the most rapid clot lysis by t-PA oc-
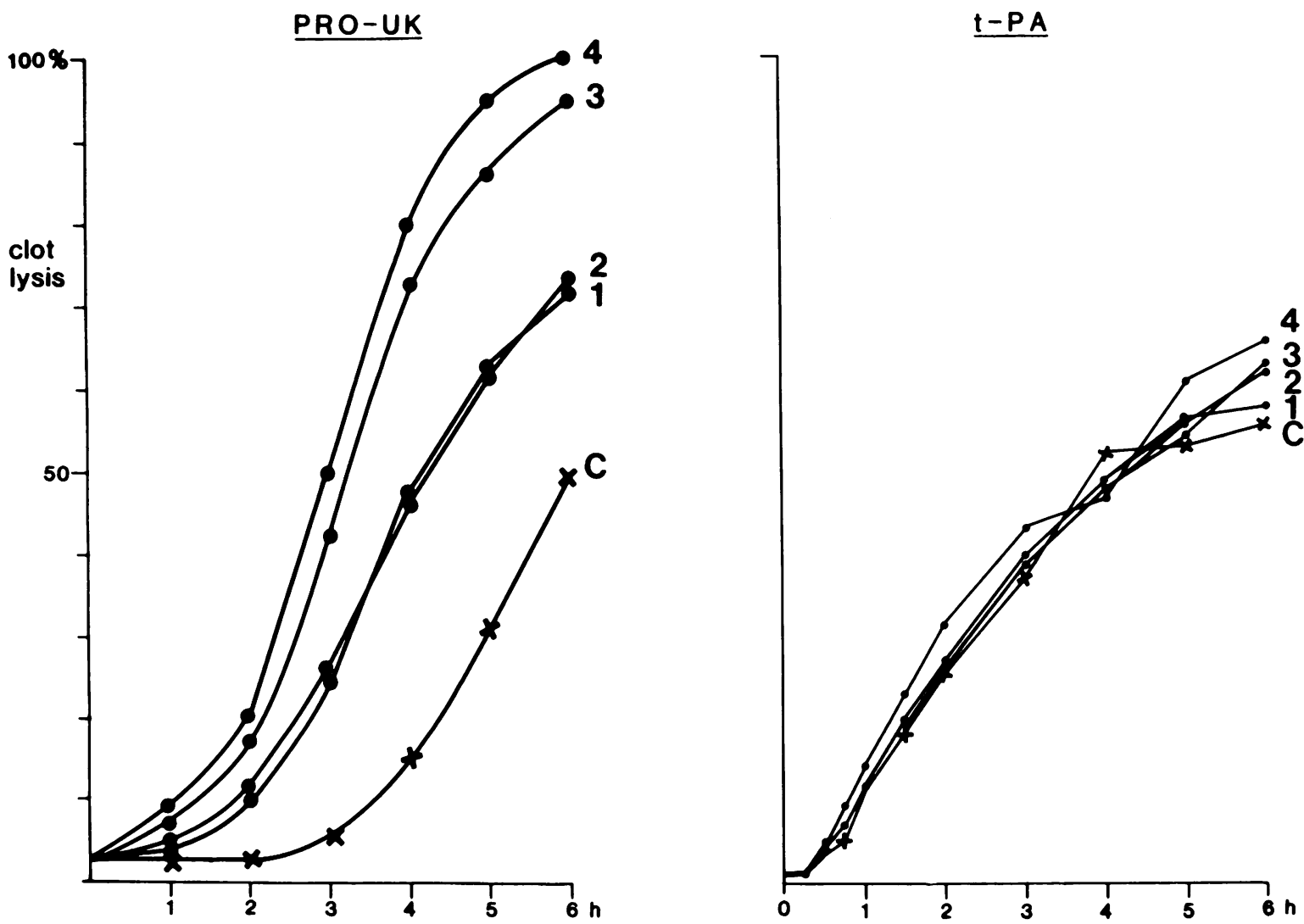

Figure 2. Effect of plasmin $(2 \mathrm{ng} / \mathrm{ml})$ pretreatment of clots on lysis induced by pro-UK $(300 \mathrm{ng} / \mathrm{ml})$ or t-PA $(50 \mathrm{ng} / \mathrm{ml})$. Time of plasmin treatment: (C) untreated control; (1) 5; (2) 10; (3) 20; (4) $30 \mathrm{~min}$. 


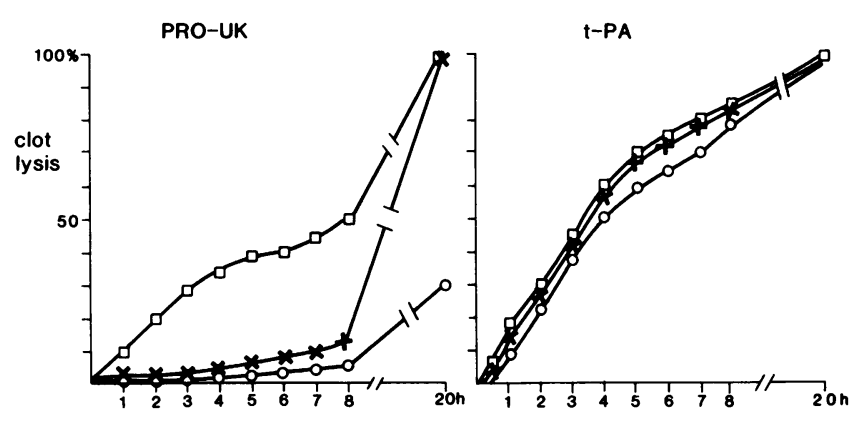

Figure 3. Reversal of the plasmin pretreatment effect by carboxypeptidase B. Lysis by pro-UK $(150 \mathrm{ng} / \mathrm{ml})$ or by t-PA $(50 \mathrm{ng} / \mathrm{ml})$ of: untreated clots $(x)$; plasmin-treated clots $(\square)$; plasmin-treated clots also treated with carboxypeptidase B (O).

curred at the beginning and was followed by a deceleration after $1-2 \mathrm{~h}$, before the fibrin substrate became limiting. In this system, specificity was lost with pro-UK $>600 \mathrm{ng} / \mathrm{ml}$ and with $\mathrm{t}-\mathrm{PA}>250 \mathrm{ng} / \mathrm{ml}$ (Fig. 5).

The combination of pro-UK and t-PA at the maximal concentrations used above produced significant plasminogen and fibrinogen losses. However, at certain combinations of the two activators, the rate of lysis (slope of the lysis curve) could be greatly enhanced without loss of specificity. Both high and low ratios of pro-UK to t-PA were examined. The optimal performance in terms of lysis rate with maintenance of specificity was obtained with a combination of a near maximal amount of pro-UK and a relatively small amount of t-PA. The peak lysis rate (slope of the steepest phase) at maximum doses was comparable for pro-UK and for t-PA ( $\sim 45 \%$ per hour) as calculated from Fig. 5. The mean lysis rate at the maximum dose was slightly greater for pro-UK $(29 \% / \mathrm{h})$ than for t-PA $(21 \% / \mathrm{h})$. By comparison, a combination of $500 \mathrm{ng} / \mathrm{ml}$ of proUK with $50 \mathrm{ng} / \mathrm{ml}$ of t-PA achieved a peak rate of $60 \%$ clot lysis per hour and a mean of $50 \%$ per hour without significant loss of either plasminogen or fibrinogen, indicating that fibrin

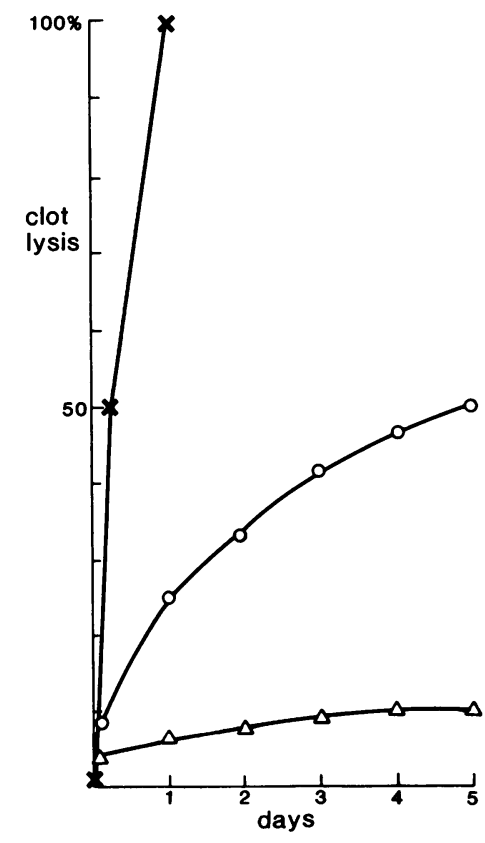

Figure 4. Lysis of carboxypeptidase B-treated clots by pro-UK, $300 \mathrm{ng} / \mathrm{ml}(\Delta)$; t-PA, $50 \mathrm{ng} / \mathrm{ml}(0)$; or by a combination of these $(x)$. selective plasminogen activation was significantly enhanced by this combination (Fig. 6).

\section{Discussion}

Clot lysis induced by pure pro-UK in the model system was characterized by an inefficient onset followed by a rapid linear rate of lysis. In contrast, clot lysis by t-PA started off briskly and then slowed to follow a less steep linear time course. The maximal rate of fibrin specific lysis by pro-UK in its second phase and of t-PA in its initial phase were comparable though the time to complete clot lysis by pro-UK was less. The combination at a high pro-UK to t-PA ratio induced clot lysis that was about twice as rapid as with either activator alone indicating that significantly more fibrin localized plasminogen activation was possible with combinations compared with either activator alone.

We have found previously that combinations of pro-UK and t-PA, at high concentration ratios $(\geq 5: 1)$, induced a greater than additive effect without compromise of specificity (9). This phenomenon was associated with attenuation of the lag phase of pro-UK-induced clot lysis by t-PA. In the present study, similar potentiation of clot lysis was obtained by the addition of a small amount of UK to pro-UK, or by pretreatment of clots with UK followed by incubation with pro-UK. In each of these experiments, the lag phase of pro-UK induced lysis was markedly attenuated.

It was previously postulated that potentiation of pro-UK induced clot lysis in these experiments was mediated by plasmic modification of fibrin, induced by the early onset of action of t-PA (9). This conclusion was supported by the present findings that pretreatment of clots with a little plasmin, suffcient only to induce $<1 \%$ clot lysis, similarly potentiated proUK-induced lysis. By contrast, plasmin pretreatment of clots did not potentiate t-PA induced clot lysis.

Harpel has observed that plasmin exposes new plasminogen binding sites on fibrin (15). Selective activation by pro-UK of fibrin bound plasminogen, in contrast to free native plasminogen, has been proposed as the mechanism responsible for the fibrin specificity of pro-UK in vitro (5). Since plasmin exposes carboxy-terminal lysine residues on fibrin, the findings implicate these terminal lysines as the plasminogen binding domains on fibrin critical to pro-UK induced clot lysis.

Plasmin degrades fibrin(ogen) by proteolytic cleavage at certain lysine and arginine residues, separating fragments $\mathrm{E}$ and $\mathrm{D}$ (or D-dimer) as soluble products and releasing terminal peptides from the $\alpha$ - and $\gamma$-chains (6). As a result, C-terminal lysines are exposed, which are binding sites for plasminogen. Plasmic fragments of fibrin(ogen) have variously been shown to bind plasminogen $(17,18)$. Christensen $(18)$ found that the binding of fragment $\mathrm{E}$ to plasminogen-Sepharose was completely abolished by treatment with carboxypeptidase B, an enzyme which specifically removes carboxy-terminal basic amino acids (19), further implicating lysine C-termini on fibrin derivatives.

In the present study, carboxypeptidase B was found to reverse the potentiating effect of plasmin on pro-UK induced clot lysis confirming that the potentiation was due to the exposure of carboxy-terminal lysines. Furthermore, carboxypeptidase B pretreatment of clots, followed by washing, greatly 

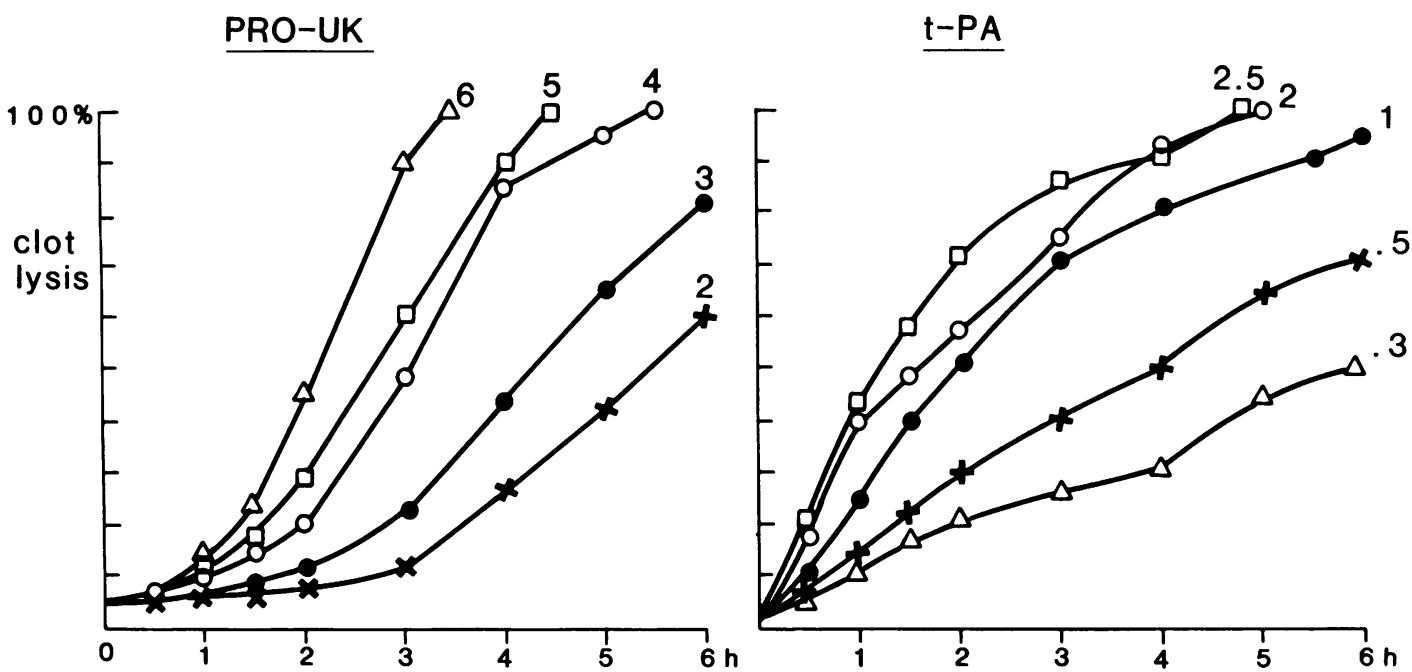

Figure 5. Lysis of fibrin clots as a function of plasminogen activator concentrations below their thresholds of specificity. Numbers at each line represent concentration in nanograms per milliliter divided by 100 .

retarded the fibrinolytic effect of pro-UK. This finding suggests that some $\mathrm{C}$-terminal lysine promotive sites were available on clots made up from fibrinogen (Kabi) that were removed by carboxypeptidase $B$. The identical carboxypeptidase B treatment of clots, however, had little effect on t-PA induced lysis. These contrasting effects on t-PA and pro-UK of plasmin or carboxypeptidase B modification of fibrin have implications relative to their respective plasminogen substrates.

A more uniform effect was observed when carboxypeptidase $\mathrm{B}$ was added to the plasma milieu during the course of clot lysis. Under these conditions, it inhibited to some extent the effect of both activators and of UK as well. This probably relates to an additional role of C-terminal lysines that bind plasmin thereby stabilizing it against inhibition by $\alpha_{2}$-antiplasmin (20). This effect on plasmin helps to sustain fibrinolysis.

Tissue plasminogen activator binds tightly to fibrin and forms a ternary complex with fibrin bound plasminogen. This mechanism is responsible for plasminogen activation and for the fibrin specificity of t-PA-induced clot lysis (1). Although, the precise binding site for t-PA on fibrin has not been identified, Niewenhuizen has shown that an internal lysine (residue A $\alpha$-Lys-157) is important for the stimulation of plasminogen activation by t-PA $(21,22)$. This conforms to the determinant for the "amino-hexyl" binding site on plasminogen kringle 5 described by Christensen (23). Our findings that neither plas-

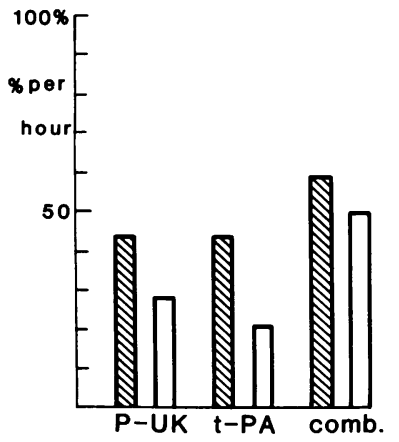

Figure 6. Rates of clot lysis (in percent per hour) for each activator just below their respective thresholds of specificity: pro-UK $(600 \mathrm{ng} / \mathrm{ml})$, t-PA $(250 \mathrm{ng} / \mathrm{ml})$; and for a combination of pro-UK $(500 \mathrm{ng} / \mathrm{ml})$ and $\mathrm{t}-\mathrm{PA}(50 \mathrm{ng} / \mathrm{ml})$. The hatched bars represent the maximal linear rates and the open bars represent the average rate over the course of time to completion. min modification of fibrin nor subsequent carboxypeptidase B treatment influenced clot lysis by t-PA are consistent. They indicate that plasminogen bound to the $\mathrm{C}$-terminal lysines was not accessible to t-PA, thereby implicating an internal lysine as the plasminogen binding site on fibrin involved in the ternary complex.

Pro-urokinase does not bind to a fibrin clot suspended in plasma (4). The present study shows that pro-UK also does not activate the plasminogen bound to the internal lysine residue which is the substrate for t-PA. This is evidenced by the initial resistance of the undegraded clot, containing no C-terminal lysines, to pro-UK induced lysis as shown by the characteristic lag phase which was abolished by plasmin. The dependence of pro-UK on the presence of terminal lysine residues suggests that this binding site, and not the internal lysines, induces the specific conformational change in plasminogen which makes it susceptible to activation by pro-UK. It has been suggested that the "pseudo-Lys"-conformation of Glu-plasminogen is responsible for the fibrin selectivity of pro-UK induced clot lysis. Fibrin specificity was lost when Glu-plasminogen in plasma was replaced by Lys-plasminogen (5). Moreover, Lysplasminogen was shown to be far more sensitive than the Gluform to activation by pro-UK (7).

In conclusion, the findings indicate that t-PA and pro-UK induce fibrinolysis by complementary mechanisms by which their apparent synergism can be explained. Fig. 7 presents schematically the proposed sequence of events. The sites required for binding of t-PA and Glu-plasminogen to form the ternary activation complex are already present on the undegraded clot that leads to early plasmin generation and degradation of the fibrin surface, opening up a new class of Glu-plasminogen binding sites. Binding at these sites conformationally modifies the Glu-plasminogen to the form emulating Lysplasminogen. It is this form that is the preferred substrate for activation by pro-UK but which appears to be inaccessible to activation by t-PA. The pro-UK may be locally converted by the formed plasmin to UK, further accelerating clot lysis. The two different activation pathways can then proceed in a complementary fashion to efficiently consume the fibrin substrate. However, in the absence of t-PA (or UK) the creation of the 

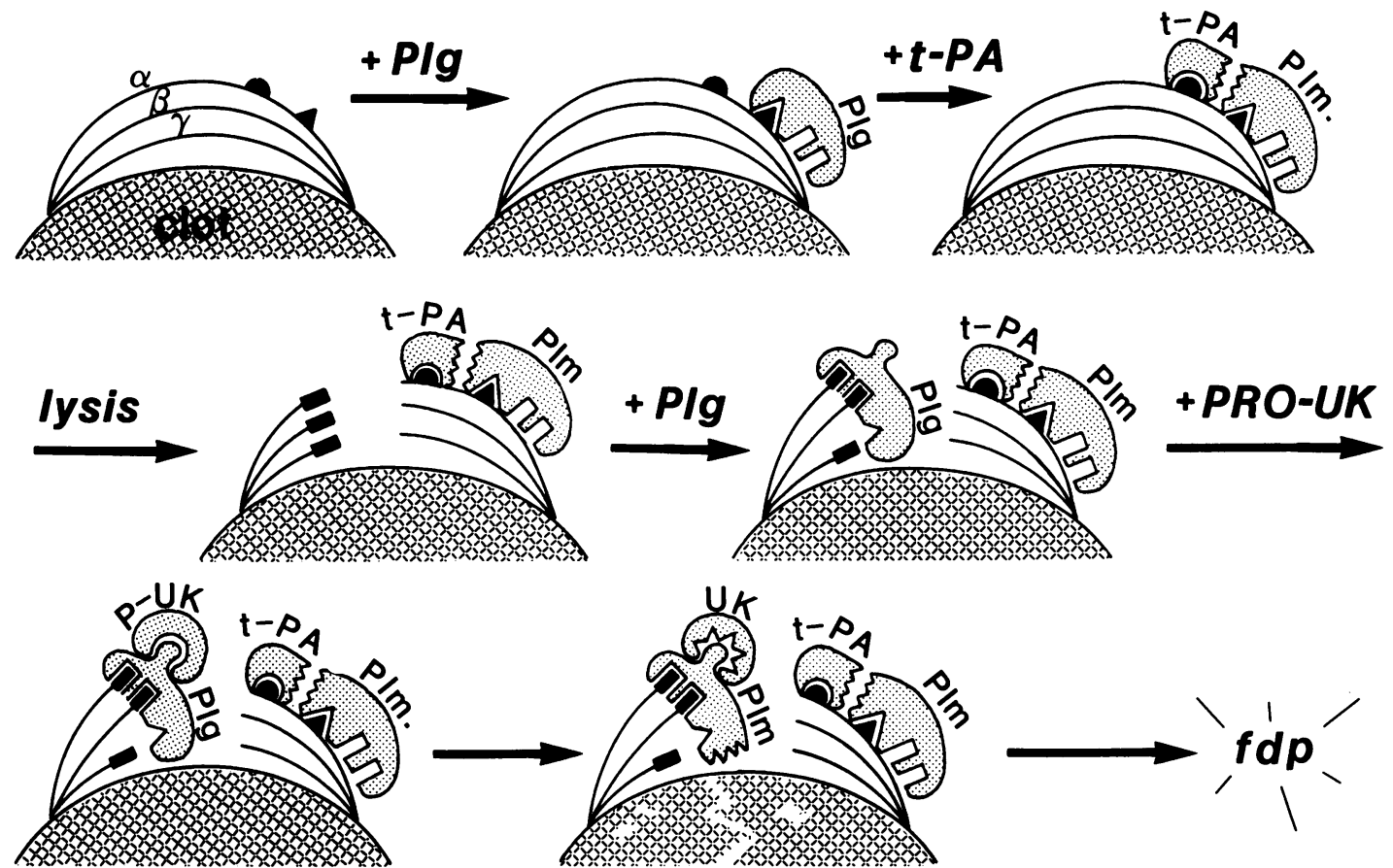

Figure 7. Schema of the proposed stages in lysis of a fibrin clot induced by t-PA and pro-UK: An undegraded clot (upper left, showing schematically the intact $\alpha-, \beta$-, and $\gamma$-chains of a half fibrin monomer) contains the internal lysine residue, for binding of Glu-plasminogen $(P l g)$, and the binding site for t-PA, necessary to form the ternary activation complex. The binding of G-Plg and t-PA results in plasmin $(P l m)$ formation and the initiation of lysis, which exposes $\mathrm{C}$ -

second class of binding sites in the first step is very slow and rate limiting, explaining the slow onset of lysis when pro-UK is used alone. This schema helps explain why t-PA and pro-UK are each relatively inefficient fibrinolytic agents by themselves, as shown in vitro. The schema is also consistent with certain in vivo findings such as the high dosages that have been found necessary for effective therapeutic thrombolysis. The present study provides an experimental basis for a more efficient clinical strategy through the use of both activators together.

\section{Acknowledgments}

Supported by National Institutes of Health grant HL-23367-08.

\section{References}

1. Hoylaerts, M., D. C. Rijken, H. R. Lijnen, and D. Collen. 1982. Kinetics of the activation of plasminogen by human tissue plasminogen activator. Role of fibrin. J. Biol. Chem. 257:2912-2919.

2. Husain, S. S., V. Gurewich, and B. Lipinski. 1981. Purification of a new high MW single chain form of urokinase (UK) from urine. Thromb. Haemostasis. 46:11. (Abstr.)

3. Husain, S. S., and V. Gurewich. 1983. Purification and partial characterization of a single chain, high molecular weight form of urokinase from human urine. Arch. Biochem. Biophys. 220:31-38.

4. Gurewich, V., and R. Pannell. 1983. The fibrin specificity of single chain-urokinase (SC-UK) induced proteolysis is not dependent on fibrin binding. Thromb. Haemostasis. 50:386.

5. Pannell, R., and V. Gurewich. 1986. Pro-urokinase-a study of terminal lysine residues on fibrin. Plasminogen binds to these termini. A conformational change in plasminogen is thereby induced providing the appropriate favored substrate for pro-UK $(P-U K)$. The plasmin thus formed probably activates pro-UK to UK thereby amplifying additional plasmin generation. The plasmin generated by the complementary actions of t-PA and pro-UK/UK lyses the fibrin clot to fibrin degradation products $(f d p)$.

its stability in plasma and of a mechanism for its selective fibrinolytic effect. Blood. 67:1215-1223.

6. Violand, B. N., J. M. Sodetz, and F. J. Castellino. 1975. The effect of epsilon aminocaproic acid on the gross conformation of plasminogen and plasmin. Arch. Biochem. Biophys. 170:300-305.

7. Pannell, R., and V. Gurewich. 1987. The activation of plasminogen by single chain urokinase or by two chain urokinase-a demonstration that single chain urokinase has a low catalytic activity (prourokinase). Blood. 69:22-26.

8. Lijnen, H. R., C. Zammarron, M. Blaber, M. E. Winkler, and D. Collen. 1986. Activation of plasminogen by pro-urokinase. I. Mechanism. J. Biol. Chem. 261:1253-1258.

9. Gurewich, V., and R. Pannell. 1986. A comparative study of the efficacy and specificity of tissue plasminogen activator and pro-urokinase. Demonstration of synergism and of different thresholds of nonselectivity. Thromb. Res. 44:217-228.

10. Gurewich, V. and R. Pannell. 1987. Synergism of tissue plasminogen activator (t-PA) and single chain urokinase-type plasminogen activator (scu-PA) on clot lysis in vitro and a mechanism for this effect. Thromb. Haemostasis. 57:372.

11. Collen, D., F. DeCock, E. Demarsin, H. R. Lijnen, and D. C. Stump. 1986. Absence of synergism between tissue type plasminogen activator (t-PA), single-chain urokinase type plasminogen activator (scu-PA) and urokinase on clot lysis in a plasma milieu in vitro. Thromb. Haemostasis. 56:35-39.

12. Collen, D., D. C. Stump, and F. Van de Werf. 1986. Coronary thrombolysis in patients with myocardial infarction by intravenous infusion of synergistic thrombolytic agents. Am. Heart J. 11:10831084.

13. Collen, D., J. Stassen, D. C. Stump, and M. Verstraete. 1986. Synergism of thrombolytic agents in vivo. Circulation. 14:838-842.

14. Gurewich, V., R. Pannell, S. Louie, P. Kelley, R. L. Suddith, 
and R. Greenlee. 1984. Effective and fibrin-specific clot lysis by a zymogen precursor form of urokinase (pro-urokinase). A study in vitro and in two animal species. J. Clin. Invest. 73:1731-1739.

15. Harpel, P. C., T.-S. Chang, and E. Verderber. 1985. Tissue plasminogen activator and urokinase mediate the binding of Glu-plasminogen to plasma Fibrin I. Evidence for new binding sites in plasmin-degraded Fibrin I. J. Biol. Chem. 260:4432-4440.

16. Budzynski, A. Z. 1986. Fibrinogen and fibrin: Biochemistry and pathophysiology. Cr. Rev. Hem. Onc. 6:97-146.

17. Varadi, A., and L. Patthy. 1984. (Leu-121-Lys-122) segment of fibrinogen is in a region essential for plasminogen binding by fibrinogen fragment E. Biochemistry. 23:2108-2112.

18. Christensen, U. 1985. C-terminal lysine residues of fibrinogen fragments essential for binding to plasminogen. FEBS (Fed. Eur. Bio chem. Soc.) Lett. 182:43-46.
19. Folk, J. E. 1970. Carboxypeptidase B. Methods Enzymol. 19:504-508.

20. Collen, D. 1980. On the regulation and control of fibrinolysis. Thromb. Haemostasis. 43:77-89.

21. Nieuwenhuizen, W., A. Vermond, M. Voskuilen, D. W. Traas, and J. H. Verheijen. 1983. Identification of a site in fibrin(ogen) which is involved in the acceleration of plasminogen activation by tissue-type plasminogen activator. Biochim. Biophys. Acta. 748:86-92.

22. Nieuwenhuizen, W., M. Voskuilen, A. Vermond, G. Veeneman, and J. V. Boom. 1986. Lysine residue $A \alpha-157$ of fibrinogen plays a crucial role in the acceleration of the plasminogen activation by tissue plasminogen activator (t-PA). Fibrinolysis. Abstract \#9.

23. Christensen, U. 1984. The AH-site of plasminogen and two C-terminal fragments: A weak lysine-binding site preferring ligands not carrying a free carboxylate function. Biochem. J. 223:413-421. 